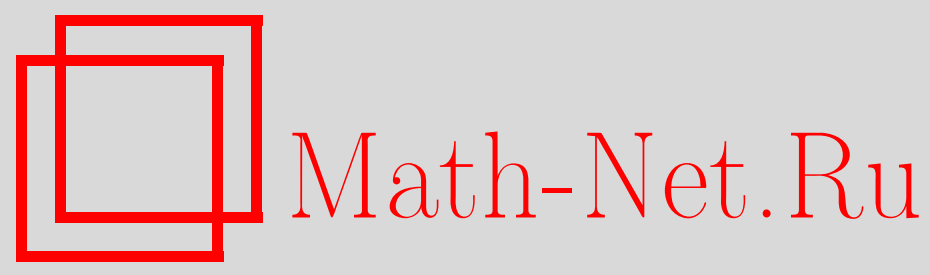

В. Г. Вердиев, Экстремальные задачи на классах полиномов и сплайнов, Матем. заметки, 1998, том 63, выпуск 4, 494502

DOI: https://doi.org/10.4213/mzm1309

Использование Общероссийского математического портала Math-Net.Ru подразумевает, что вы прочитали и согласны с пользовательским соглашением http://www. mathnet.ru/rus/agreement

Параметры загрузки:

IP : 54.162.85.209

26 апреля 2023 г., 12:38:48

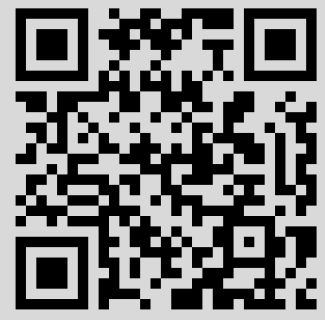




\section{ЭКСТРЕМАЛЬНЫЕ ЗАДАЧИ}

\section{НА КЛАССАХ ПОЛИНОМОВ И СПЛАЙНОВ}

\section{В.Г. Вердиев}

Методами теории экстремальных задач доказаны теоремы о существовании функций с заданной последовательностью экстремальных значений.

Библиографоия: 24 названия.

1. Введение. В статье рассматривается один достаточно общий метод доказательства теорем о существовании функций с заданными значениями экстремумов, основанный на средствах теории экстремальных задач [1]-[3].

Пусть $\Delta:=[0,1] ; \mathfrak{P}_{N}-$ совокупность алгебраических полиномов степени $N ; S_{m}(\Delta$, $R_{n}$ ) - совокупность сплайнов порядка $m$ с узлами $\left\{t_{i}\right\}_{i=1}^{n}$, образующими разбиение $R_{n}$ отрезка $\Delta$, дефекта 1 . В дальнейшем любую последовательность $\xi=\left\{\xi_{i}\right\}_{i=0}^{N}$, члены которой удовлетворяют неравенствам $(-1)^{i+1}\left(\xi_{i+1}-\xi_{i}\right)<0,0 \leqslant i \leqslant N-1$, будем назьвать последовательностью әкстремальных значений.

ТЕОРемА 1. Для любой заданной последовательности әкстремальных значений $\xi=\left\{\xi_{i}\right\}_{i=0}^{N}$ существует единственный алгебраический полином $\widehat{P}(\cdot) \in \mathfrak{P}_{N} u$ единственная система из $N+1$ точек $\left\{\widehat{\tau}_{i}\right\}_{i=0}^{N} \subset \Delta$, удовлетворяющие условиям

$$
\begin{gathered}
\widehat{P}\left(\widehat{\tau}_{i}\right)=\xi_{i}, \quad 0 \leqslant i \leqslant N, \\
\widehat{P}^{\prime}\left(\widehat{\tau}_{i}\right)=0, \quad 1 \leqslant i \leqslant N-1, \\
0=: \widehat{\tau}_{0}<\widehat{\tau}_{1}<\cdots<\widehat{\tau}_{N-1}<\widehat{\tau}_{N}:=1 .
\end{gathered}
$$

Теорема 2. Пусть $m \in \mathbb{N}, n \in \mathbb{N}, N \in \mathbb{N} u m+n=N$. Тогда для любой заданной последовательности экстремальных значений $\xi=\left\{\xi_{i}\right\}_{i=0}^{N}$ существует единственный сплайн $\widehat{x}(\cdot) \in S_{m}\left(\Delta, R_{n}\right)$ и единственная система из $N+1$ точек $\left\{\widehat{\tau}_{i}\right\}_{i=0}^{N} \subset \Delta$, удовлетворяющие условиям

$$
\begin{gathered}
\widehat{x}\left(\widehat{\tau}_{i}\right)=\xi_{i}, \quad 0 \leqslant i \leqslant N, \\
\widehat{x}^{\prime}\left(\widehat{\tau}_{i}\right)=0, \quad 1<i<N-1, \\
\widehat{\tau}_{i}<t_{i}<\widehat{\tau}_{m+i}, \quad 1 \leqslant i \leqslant n, \\
0=: \widehat{\tau}_{0}<\widehat{\tau}_{1}<\cdots<\widehat{\tau}_{N-1}<\widehat{\tau}_{N}:=1 .
\end{gathered}
$$


2. История вопроса. К теореме 1 привели Ч. Дэйвиса [4], [5] исследования А. Кемпнера [6], [7], а Я. Мьщельского и С. Пашковского [8], [9] - методы построения алгебраического полинома наилучшего приближения, и они доказали ее топологическими методами. Для построения полиномов, удовлетворяющих условиям теоремы 1, В. Я. Каммерер [10] использовал идеи теории приближений, В. С. Виденский [11]-[14] и Ж. Кудела [15] применили метод последовательных приближений. Теорема 2 является распространением теоремы 1 на сплайны дефекта 1, которое осуществила М. Б. Коробкова [16]. Р. С. Джонсон [17], используя метод неподвижной точки, получил родственную теорему для моносплайнов.

3. Постановка экстремальных задач. Пусть $\Sigma^{N-1}:=\left\{\eta=\left(\eta_{1}, \ldots, \eta_{N-1}\right) \mid\right.$ $\left.\eta_{i} \geqslant 0, \sum_{i=1}^{N-1} \eta_{i}=1\right\}-(N-1)$-мерный симплекс и $\Sigma_{R_{n}}^{N-1}:=\left\{\eta^{\prime}=\left(\eta_{1}^{\prime}, \ldots, \eta_{N-1}^{\prime}\right) \mid\right.$ $\left.\eta_{j}^{\prime} \geqslant 0, \sum_{j=1}^{N-1} \eta_{j}^{\prime}=1, \sum_{j=1}^{i} \eta_{j}^{\prime}<t_{i}<\sum_{j=1}^{m+i} \eta_{j}^{\prime}, 1 \leqslant i \leqslant n, t_{i} \in\left\{t_{i}\right\}_{i=1}^{n}=: R_{n}\right\}$, $\tau(\eta):=\left(\tau_{0}(\eta), \tau_{1}(\eta), \ldots, \tau_{N}(\eta)\right)$ и $\tau^{\prime}\left(\eta^{\prime}\right):=\left(\tau_{0}^{\prime}\left(\eta^{\prime}\right), \tau_{1}^{\prime}\left(\eta^{\prime}\right), \ldots, \tau_{N}^{\prime}\left(\eta^{\prime}\right)\right)$, где

$$
\begin{array}{cc}
\tau_{0}(\eta):=0, \quad \tau_{i}(\eta)=\sum_{j=1}^{i} \eta_{j}, \quad 1 \leqslant i \leqslant N-1, \quad \tau_{N}(\eta):=1 \\
\tau_{0}^{\prime}\left(\eta^{\prime}\right):=0, \quad \tau_{i}^{\prime}\left(\eta^{\prime}\right)=\sum_{j=1}^{i} \eta_{j}^{\prime}, \quad 1 \leqslant i \leqslant N-1, \quad \tau_{N}^{\prime}\left(\eta^{\prime}\right):=1 .
\end{array}
$$

Как обычно, $\partial \Sigma^{N-1}$ и $\partial \Sigma_{R_{n}}^{N-1}$-гранищы, int $\Sigma^{N-1}$ и int $\Sigma_{R_{n}}^{N-1}$ - множества внутренних точек $\Sigma^{N-1}$ и $\Sigma_{R_{n}}^{N-1}$ соответственно. Ясно, что при любых $\eta \in \operatorname{int} \Sigma^{N-1}$ и $\eta^{\prime} \in \Sigma_{R_{n}}^{N-1}$ имеем

$$
\begin{gathered}
0=: \tau_{0}(\eta)<\tau_{1}(\eta)<\cdots<\tau_{N}(\eta):=1, \\
0=: \tau_{0}^{\prime}\left(\eta^{\prime}\right)<\tau_{1}^{\prime}\left(\eta^{\prime}\right)<\cdots<\tau_{N}^{\prime}\left(\eta^{\prime}\right):=1 .
\end{gathered}
$$

Зафиксируем произвольным образом некоторую последовательность экстремальных значений $\xi=\left\{\xi_{i}\right\}_{i=0}^{N}$. Тогда для любых $\eta \in \operatorname{int} \Sigma^{N-1}$ и $\eta^{\prime} \in \Sigma_{R_{n}}^{N-1}$ согласно интерполяционной формуле Лагранжа существуют единственный полином $P(\cdot, \eta) \in \mathfrak{P}_{N}$ и по теореме $1.3\left[18\right.$, с. 27] единственньй сплайн $x\left(\cdot, \eta^{\prime}\right) \in S_{m}\left(\Delta, R_{n}\right)$ такие, что

$$
P\left(\tau_{i}(\eta), \eta\right)=\xi_{i}, \quad x\left(\tau_{i}^{\prime}\left(\eta^{\prime}\right), \eta^{\prime}\right)=\xi_{i}, \quad 0 \leqslant i \leqslant N .
$$

Положим

$$
\begin{gathered}
\mathfrak{P}\left(\operatorname{int} \Sigma^{N-1}, \xi\right):=\left\{P(\cdot, \eta) \in \mathfrak{P}_{N} \mid P\left(\tau_{i}(\eta), \eta\right)=\xi_{i}, \eta \in \operatorname{int} \Sigma^{N-1}, \xi_{i} \in \xi\right\}, \\
S\left(\operatorname{int} \Sigma_{R_{n}}^{N-1}, \xi\right):=\left\{x\left(\cdot, \eta^{\prime}\right) \in S_{m}\left(\Delta, R_{n}\right) \mid x\left(\tau_{i}^{\prime}\left(\eta^{\prime}\right), \eta^{\prime}\right)=\xi_{i}, \eta^{\prime} \in \Sigma^{N-1}, \xi_{i} \in \xi\right\} .
\end{gathered}
$$

Рассмотрим вариационные задачи.

ЗАДАчА 1. Найти

$$
\lambda_{N}(\xi)= \begin{cases}\inf P(2, \eta) & \text { при четном } N \\ \sup P(2, \eta) & \text { при нечетном } N\end{cases}
$$

если $\eta \in \operatorname{int} \Sigma^{N-1}$. 
ЗАДАчА 2. Найти

$$
\lambda_{N}^{\prime}(\xi)= \begin{cases}\inf x(2, \eta) & \text { при четном } N \\ \sup x(2, \eta) & \text { при нечетном } N\end{cases}
$$

если $\eta^{\prime} \in \Sigma_{R_{n}}^{N-1}$.

\section{4. Существование решений экстремальных задач.}

Лемма 1. Решение задачи 1 существует.

ДокАЗАтЕльство. Пусть $\eta \in \operatorname{int} \Sigma^{N-1}$ и $\bar{\eta} \in \partial \Sigma^{N-1}$. Покажем, что абсолютное значение $|P(2, \eta)|$ при $t=2$ полинома $P(t, \eta) \in \mathfrak{P}\left(\right.$ int $\left.\Sigma^{N-1}, \xi\right)$ стремится к $+\infty$ при $\eta \rightarrow \bar{\eta}$. Точка $\bar{\eta} \in \partial \Sigma^{N-1}$, поэтому некоторые из ее координат $\bar{\eta}_{1}, \bar{\eta}_{2}, \ldots, \bar{\eta}_{N-1}$ равны 0 . Совокупность индексов, соответствующих нулевым координатам точки $\bar{\eta} \in \partial \Sigma^{N-1}$, обозначим через $I$, т.е. $i \in I$, если $\bar{\eta}_{i}=0$, и $i \notin I$, если $\bar{\eta}_{i}>0$. Рассуждения проведем для $I=\{1\}$. В остальных случаях рассуждения аналогичны. Тогда $\bar{\eta}=\left(0, \bar{\eta}_{2}, \ldots\right.$, $\left.\bar{\eta}_{N-1}\right) \in \partial \Sigma^{N-1}$ соответствует точка $\bar{\tau}=\left(0,0, \bar{\tau}_{2}, \ldots, \bar{\tau}_{N}\right)$, координаты которой удовлетворяют неравенствам $0<\bar{\tau}_{2}<\cdots<\bar{\tau}_{N}=1$. Полином $P(t, \eta) \in \mathfrak{P}\left(\operatorname{int} \Sigma^{N-1}, \xi\right)$, соответствующий точке $\eta$, представим в виде суммы $P(t, \eta)=P_{1}(t, \eta)+P_{2}(t, \eta)$ двух полиномов $P_{1}(t, \eta) \in \mathfrak{P}_{N}$ и $P_{2}(t, \eta) \in \mathfrak{P}_{N}$ таких, что

$$
\begin{array}{cl}
P_{1}(0, \eta)=\xi_{0}, \quad P_{1}\left(\tau_{1}, \eta\right)=\xi_{1}, & P_{1}\left(\tau_{i}, \eta\right)=0, \\
P_{2}(0, \eta)=P_{2}\left(\tau_{1}, \eta\right)=0, & P_{2}\left(\tau_{i}, \eta\right)=\xi_{i},
\end{array}
$$

Как несложно показать, при $\eta \rightarrow \bar{\eta}$ полином $P_{2}(t, \eta)$ преобразуется в алгебраический полином $\bar{P}_{2}(t) \in \mathfrak{P}_{N}$, при этом

$$
0=\bar{P}_{2}(0)=\bar{P}_{2}^{\prime}(0), \quad \bar{P}_{2}^{\prime}\left(\bar{\tau}_{i}\right)=\xi_{i}, \quad 2 \leqslant i \leqslant N .
$$

Используя интерполяционную формулу Лагранжа, запишем полином $P_{1}(t, \eta)$ в виде $P_{1}(t, \eta)=\xi_{0} Q_{0}(t, \eta)+\xi_{1} Q_{1}(t, \eta)$, где

$$
Q_{0}(t, \eta)=\frac{\left(t-\tau_{1}\right) \cdots\left(t-\tau_{N}\right)}{\left(-\tau_{1}\right) \cdots\left(-\tau_{N}\right)}, \quad Q_{1}(t, \eta)=\frac{t\left(t-\tau_{2}\right) \cdots\left(t-\tau_{N}\right)}{\tau_{1}\left(\tau_{1}-\tau_{2}\right) \cdots\left(\tau_{1}-\tau_{N}\right)}
$$

Учитьвая, что

$$
P(2, \eta)=Q_{0}(2, \eta)\left(\xi_{0}+\xi_{1} \frac{Q_{1}(2, \eta)}{Q_{0}(2, \eta)}\right)+P_{2}(2, \eta)
$$

в силу равенств

$$
\lim _{\eta \rightarrow \bar{\eta}} \tau=\bar{\tau}, \quad \lim _{\eta \rightarrow \bar{\eta}} Q_{0}(t, \eta)= \pm \infty, \quad \lim _{\eta \rightarrow \bar{\eta}} \frac{Q_{1}(2, \eta)}{Q_{0}(2, \eta)}=-1
$$

имеем

$$
\lim _{\eta \rightarrow \bar{\eta}}|P(2, \eta)|=+\infty
$$

Следовательно, при любом $\bar{\eta} \in \partial \Sigma^{N-1}$ непрерывная на int $\Sigma^{N-1}$ функция $|P(2, \eta)|$ от $\eta$ такова, что выполняется (3). Поэтому согласно следствию из теоремы Вейерштрасса [2, c. 24] в int $\Sigma^{N-1}$ существует точка $\widehat{\eta}$, являющаяся решением задачи 1 . Лемма 1 доказана. 


\section{Лемма 2. Решение задачи 2 существует.}

ДокАЗАтЕльство. Гранища множества $\Sigma_{R_{n}}^{N-1}$ состоит из точек границы $\partial \Sigma^{N-1}$ симплекса $\Sigma^{N-1}$ и точек множества $\partial \Sigma_{R_{n}}$, координаты которых определяются соотношениями

$$
\begin{gathered}
\sum_{j=1}^{i} \bar{\eta}_{j}^{\prime}=t_{i}, \quad \sum_{j=1}^{m+i} \bar{\eta}_{j}^{\prime}=t_{i}, \quad 1 \leqslant i \leqslant n, \quad t_{i} \in\left\{t_{i}\right\}_{i=1}^{n}=R_{n} \\
\bar{\eta}_{i}^{\prime}>0, \quad 1 \leqslant i \leqslant N-1, \quad \sum_{i=1}^{N-1} \bar{\eta}_{i}^{\prime}=1 .
\end{gathered}
$$

Покажем, что абсолютное значение $\left|x\left(2, \eta^{\prime}\right)\right|$ сплайна $x\left(\cdot, \eta^{\prime}\right) \in S\left(\right.$ int $\left.\Sigma_{R_{n}}^{N-1}, \xi\right)$, соответствуюшего $\eta^{\prime} \in \operatorname{int} \Sigma_{R_{n}}^{N-1}$, в точке $t=2$ стремится к $+\infty$ при $\eta^{\prime} \rightarrow \bar{\eta}^{\prime} \in \partial \Sigma_{R_{n}}^{N-1}$.

Для точек $\left\{\tau_{i}^{\prime}\right\}_{i=0}^{N}$ системы $\tau^{\prime}=\left(\tau_{0}^{\prime}, \tau_{1}^{\prime}, \ldots, \tau_{N}^{\prime}\right)$, построенной по произвольно фиксированной точке $\eta^{\prime} \in \operatorname{int} \Sigma_{R_{n}}^{N-1}$, и точек $\left\{t_{i}\right\}_{i=1}^{n}$ разбиения $R_{n}$ имеем

$$
\begin{gathered}
0=: \tau_{0}^{\prime}<\tau_{1}^{\prime}<\cdots<\tau_{N-1}^{\prime}<\tau_{N}^{\prime}:=1, \\
\tau_{i-1}^{\prime}<t_{i}<\tau_{i+n}^{\prime}, \quad 1 \leqslant i \leqslant n,
\end{gathered}
$$

согласно определению множества $\Sigma_{R_{n}}^{N-1}$. Неравенства (4) служат необходимьм и достаточным условием существования единственного сплайна [19] в $S_{m}\left(\Delta, R_{n}\right)$, принимающего значение $\xi_{i}$ в точке $\tau_{i}^{\prime}, 0 \leqslant i \leqslant N$, и для этого сплайна имеет место интерполяционная формула Лагранжа (см. $\left[18\right.$, с. 29]). Таким сплайном является $x\left(\cdot, \eta^{\prime}\right) \in$ $S\left(\right.$ int $\left.\Sigma_{R_{n}}^{N-1}, \xi\right)$, поэтому запишем его следуюшим образом:

$$
x\left(t, \eta^{\prime}\right)=\sum_{i=0}^{N} \xi_{i} \frac{S\left(\tau_{0}^{\prime}, \tau_{1}^{\prime}, \ldots, \tau_{i-1}^{\prime}, t, \tau_{i+1}^{\prime}, \ldots, \tau_{N}^{\prime}\right)}{S\left(\tau_{0}^{\prime}, \tau_{1}^{\prime}, \ldots, \tau_{N}^{\prime}\right)},
$$

где

$$
\begin{aligned}
& S\left(\tau_{0}^{\prime}, \tau_{1}^{\prime}, \ldots, \tau_{i-1}^{\prime}, t, \tau_{i+1}^{\prime}, \ldots, \tau_{N}^{\prime}\right)
\end{aligned}
$$

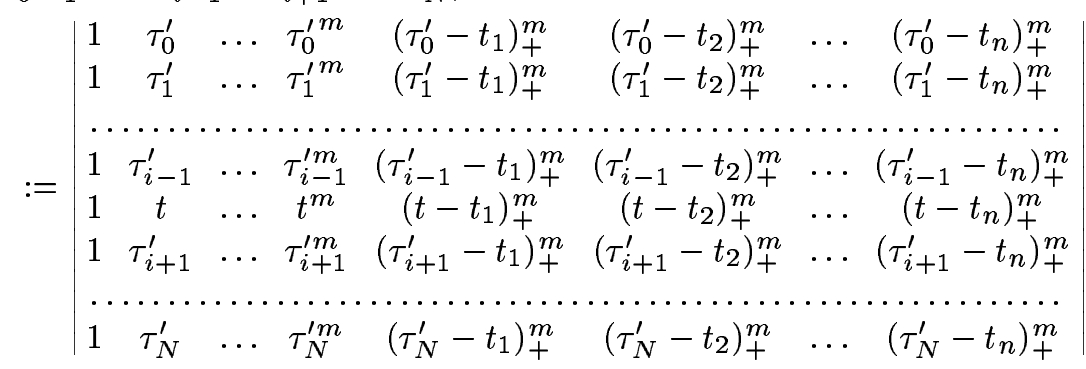

и $S\left(\tau_{0}^{\prime}, \tau_{1}^{\prime}, \ldots, \tau_{N}^{\prime}\right):=S\left(\tau_{0}^{\prime}, \tau_{1}^{\prime}, \ldots, \tau_{i-1}^{\prime}, \tau_{i}^{\prime}, \tau_{i+1}^{\prime}, \ldots, \tau_{N}^{\prime}\right)$, причем $S\left(\tau_{0}^{\prime}, \tau_{1}^{\prime}, \ldots, \tau_{N}^{\prime}\right)>0$,

$$
\begin{gathered}
S\left(\tau_{0}^{\prime}, \tau_{1}^{\prime}, \ldots, \tau_{i-1}^{\prime}, \tau_{j}^{\prime}, \tau_{i+1}^{\prime}, \ldots, \tau_{N}^{\prime}\right)=0 \text { при } i \neq j, 0 \leqslant i, j \leqslant N \\
S\left(\tau_{0}^{\prime}, \tau_{1}^{\prime}, \ldots, \tau_{i-2}^{\prime}, t_{i}, \tau_{i}^{\prime}, \ldots, \tau_{N}^{\prime}\right)=S\left(\tau_{0}^{\prime}, \tau_{1}^{\prime}, \ldots, \tau_{i+m-1}^{\prime}, t_{i}, \tau_{i+m+1}^{\prime}, \ldots, \tau_{N}^{\prime}\right)=0 \\
\text { при } 1 \leqslant i \leqslant n .
\end{gathered}
$$


Тем самьм, обоснована корректность определения множества сплайнов $S\left(\right.$ int $\left.\Sigma_{R_{n}}^{N-1}, \xi\right)$.

Пусть $\bar{\eta}^{\prime} \in \partial \Sigma^{N-1}$ и совокупность индексов, соответствующих нулевым координатам точки $\bar{\eta}^{\prime}$, обозначим через $I^{\prime}$. Рассмотрим случай $I^{\prime}=\{1\}$. В остальных рассуждения аналогичны. Тогда $\bar{\eta}^{\prime}=\left(0, \bar{\eta}_{2}^{\prime}, \ldots, \bar{\eta}_{N-1}^{\prime}\right) \in \partial \Sigma^{N-1}$ соответствует система точек $\bar{\tau}^{\prime}=\left(0,0, \bar{\tau}_{2}^{\prime}, \ldots, \bar{\tau}_{N}^{\prime}\right), 0<\bar{\tau}_{2}^{\prime}<\cdots<\bar{\tau}_{N}^{\prime}=1$. Сплайн $x\left(\cdot, \eta^{\prime}\right) \in S\left(\operatorname{int} \Sigma_{R_{n}}^{N-1}, \xi\right)$, соответствующий точке $\eta^{\prime} \in \operatorname{int} \Sigma_{R_{n}}^{N-1}$, с помощью интерполяционной формулы Лагранжа для сплайнов представим в виде суммы $x\left(t, \eta^{\prime}\right)=x_{1}\left(t, \eta^{\prime}\right)+x_{2}\left(t, \eta^{\prime}\right)$ двух сплайнов $x_{1}\left(\cdot, \eta^{\prime}\right) \in S_{m}\left(\Delta, R_{n}\right)$ и $x_{2}\left(\cdot, \eta^{\prime}\right) \in S_{m}\left(\Delta, R_{n}\right)$ таких, что

$$
\begin{array}{cl}
x_{1}\left(0, \eta^{\prime}\right)=\xi_{0}, \quad x_{1}\left(\tau_{1}^{\prime}, \eta^{\prime}\right)=\xi_{1}, & x_{1}\left(\tau_{i}^{\prime}, \eta^{\prime}\right)=0, \quad 2 \leqslant i \leqslant N, \\
x_{2}\left(0, \eta^{\prime}\right)=x_{2}\left(\tau_{1}^{\prime}, \eta^{\prime}\right)=0, & x_{2}\left(\tau_{i}^{\prime}, \eta^{\prime}\right)=\xi_{i},
\end{array}
$$

Заметим, что при $\eta^{\prime} \rightarrow \bar{\eta}^{\prime}$ сплайн $x_{2}\left(\cdot, \eta^{\prime}\right)$ преобразуется в сплайн $\bar{x}_{2}(\cdot) \in S_{m}\left(\Delta, R_{n}\right)$, причем

$$
\bar{x}_{2}(0)=\bar{x}_{2}^{\prime}(0)=0, \quad \bar{x}_{2}\left(\bar{\tau}_{i}^{\prime}\right)=\xi_{i}, \quad 2 \leqslant i \leqslant N .
$$

Используя равенство $(5)$, запишем сплайн $x_{1}\left(t, \eta^{\prime}\right)$ в виде

$$
x_{1}\left(t, \eta^{\prime}\right)=\frac{1}{S\left(\tau_{0}^{\prime}, \tau_{1}^{\prime}, \ldots, \tau_{N}^{\prime}\right)}\left(\xi_{0} S\left(t, \tau_{1}^{\prime}, \ldots, \tau_{N}^{\prime}\right)+\xi_{1} S\left(\tau_{0}^{\prime}, t, \ldots, \tau_{N}^{\prime}\right)\right) .
$$

Тогда для значения $x\left(2, \eta^{\prime}\right)$ сплайна $x\left(\cdot, \eta^{\prime}\right)$ в точке $t=2$ имеем

$$
x\left(2, \eta^{\prime}\right)=\frac{S\left(2, \tau_{1}^{\prime}, \ldots, \tau_{N}^{\prime}\right)}{S\left(\tau_{0}^{\prime}, \tau_{1}^{\prime}, \ldots, \tau_{N}^{\prime}\right)}\left(\xi_{0}+\xi_{1} \frac{S\left(\tau_{0}^{\prime}, 2, \ldots, \tau_{N}^{\prime}\right)}{S\left(2, \tau_{1}^{\prime}, \ldots, \tau_{N}^{\prime}\right)}\right)+x_{2}\left(2, \eta^{\prime}\right),
$$

отсюда ввиду равенств

$$
\lim _{\eta^{\prime} \rightarrow \bar{\eta}^{\prime}} \tau^{\prime}=\bar{\tau}^{\prime}, \quad \lim _{\eta^{\prime} \rightarrow \bar{\eta}^{\prime}} \frac{S\left(2, \tau_{1}^{\prime}, \ldots, \tau_{N}^{\prime}\right)}{S\left(\tau_{0}^{\prime}, \tau_{1}^{\prime}, \ldots, \tau_{N}^{\prime}\right)}=+\infty, \quad \lim _{\eta^{\prime} \rightarrow \bar{\eta}^{\prime}} \frac{S\left(\tau_{0}^{\prime}, 2, \ldots, \tau_{N}^{\prime}\right)}{S\left(2, \tau_{1}^{\prime}, \ldots, \tau_{N}^{\prime}\right)}=-1
$$

вытекает, что

$$
\lim _{\eta^{\prime} \rightarrow \bar{\eta}^{\prime} \in \partial \Sigma^{N-1}}\left|x\left(2, \eta^{\prime}\right)\right|=+\infty .
$$

Теперь пусть $\bar{\eta}^{\prime} \in \partial \Sigma_{R_{n}}$. Разберем случай, когда точка $\bar{\eta}^{\prime} \in \partial \Sigma_{R_{n}}$ имеет координаты $\bar{\eta}^{\prime}=\left(t_{1}, \bar{\eta}_{2}^{\prime}, \ldots, \bar{\eta}_{N-1}^{\prime}\right), t_{1} \in\left\{t_{i}\right\}_{i=1}^{n}=R_{n}$. В других случаях рассуждения аналогичны. Тогда $\bar{\eta}^{\prime}=\left(t_{1}, \bar{\eta}_{2}^{\prime}, \ldots, \bar{\eta}_{N-1}^{\prime}\right) \in \partial \Sigma_{R_{n}}$ соответствует система точек $\bar{\tau}^{\prime}=\left(\bar{\tau}_{0}^{\prime}, t_{1}, \bar{\tau}_{2}^{\prime}, \ldots, \bar{\tau}_{N}^{\prime}\right), 0=\bar{\tau}_{0}^{\prime}<t_{1}<\bar{\tau}_{2}^{\prime}<\cdots<\bar{\tau}_{N}^{\prime}=1$. Если $\eta^{\prime} \rightarrow \bar{\eta}^{\prime} \in \partial \Sigma_{R_{n}}$ при $\eta^{\prime} \in \operatorname{int} \Sigma_{R_{n}}^{N-1}$, то

$$
\lim _{\eta^{\prime} \rightarrow \bar{\eta}^{\prime}} \tau^{\prime}=\bar{\tau}^{\prime}, \quad \lim _{\eta^{\prime} \rightarrow \bar{\eta}^{\prime} \in \partial \Sigma_{R_{n}}} \frac{S\left(2, \tau_{1}^{\prime}, \ldots, \tau_{N}^{\prime}\right)}{S\left(\tau_{0}^{\prime}, \tau_{1}^{\prime}, \ldots, \tau_{N}^{\prime}\right)}=+\infty,
$$

поэтому из (6) следует

$$
\lim _{\eta^{\prime} \rightarrow \bar{\eta}^{\prime} \in \partial \Sigma_{R_{n}}}\left|x\left(2, \eta^{\prime}\right)\right|=+\infty .
$$

Таким образом, мы получили, что

$$
\lim _{\eta^{\prime} \rightarrow \bar{\eta}^{\prime} \in \partial \Sigma_{R_{n}}^{N-1}}\left|x\left(2, \eta^{\prime}\right)\right|=+\infty .
$$

Поэтому согласно следствию из теоремы Вейерштрасса [20, с. 24] в int $\Sigma_{R_{n}}^{N-1}$ существует точка $\widehat{\eta}$, которая является решением задачи 2 . Лемма 2 доказана. 


\section{5. Доказательства теорем.}

ДоКАЗАТЕЛЬСТВо ТЕОРЕМЫ 1 . Исходя из решения $\widehat{\eta}=\left(\widehat{\eta}_{1}, \widehat{\eta}_{2}, \ldots, \widehat{\eta}_{N-1}\right)$ задачи 1 , строим систему из $N+1$ точек $\widehat{\tau}=\left(\tau_{0}(\widehat{\eta}), \tau_{1}(\widehat{\eta}), \ldots, \tau_{N}(\widehat{\eta})\right)=:\left(\widehat{\tau}_{0}, \widehat{\tau}_{1}, \ldots, \widehat{\tau}_{N}\right)$ и полином $P(\cdot, \widehat{\eta}) \in \mathfrak{P}_{N}$. Система точек $\left(\widehat{\tau}_{0}, \widehat{\tau}_{1}, \ldots, \widehat{\tau}_{N}\right)$ и полином $P(\cdot, \widehat{\eta})$ удовлетворяют условиям теоремы 1 . Действительно, в силу построения $\widehat{\tau}$ и $P(\cdot, \widehat{\eta})$ имеем

$$
\begin{gathered}
0=: \widehat{\tau}_{0}<\widehat{\tau}_{1}<\cdots<\widehat{\tau}_{N-1}<\widehat{\tau}_{N}:=1, \\
P\left(\widehat{\tau}_{i}, \widehat{\eta}\right)=\xi_{i}, \quad 0 \leqslant i \leqslant N,
\end{gathered}
$$

так как $\widehat{\eta} \in \operatorname{int} \Sigma^{N-1}$ и $\widehat{P}(\cdot, \widehat{\eta}) \in \mathfrak{P}\left(\operatorname{int} \Sigma^{N-1}, \xi\right)$. Далее предположим, что при некотором $i_{0}, 1<i_{0}<N$, в точке $\widehat{\tau}_{i_{0}}$ для $\widehat{P}(\cdot, \widehat{\eta})$ равенство (1) нарушено, т.е.

$$
\widehat{P}^{\prime}\left(\widehat{\tau}_{i_{0}}, \widehat{\eta}\right) \neq 0 \text {. }
$$

Рассмотрим полином степени $N$

$$
\widetilde{P}(t):=\frac{\prod_{i=0}^{N}\left(t-\widetilde{\tau}_{i}\right)}{t-\widetilde{\tau}_{i_{0}}},
$$

у которого все $N$ нулей расположены на отрезке $\Delta$ и поэтому значение $\widetilde{P}(2)$ полинома $\widetilde{P}(t)$ в точке $t=2$ отлично от 0 , т.е. $\widetilde{P}(2) \neq 0$. Тогда согласно классической теореме о неявной функции $[21$, c. 166$]$ существуют $\varepsilon_{0}>0$ и отображение $T(\varepsilon)$ класса $C^{1}\left[-\varepsilon_{0}, \varepsilon_{0}\right]$ такие, что $T(0)=\widehat{\tau}_{i_{0}}$ и $\widehat{P}(T(\varepsilon) \widehat{\eta})+\varepsilon \widetilde{P}(T(\varepsilon))=\xi_{i_{0}}$ при любом $\varepsilon \in\left(-\varepsilon_{0}, \varepsilon_{0}\right)$. Отсюда следует, что при любом $\varepsilon \in\left(-\varepsilon_{0}, \varepsilon_{0}\right)$ полином $\Phi(t, \varepsilon):=\widehat{P}(t, \widehat{\eta})+\varepsilon \widetilde{P}(t) \in \mathfrak{P}\left(\right.$ int $\left.\Sigma^{N-1}, \xi\right)$. Рассмотрим значение полинома $\Phi(t, \varepsilon)$ в точке $t=2$. Сначала заметим, что

$$
\begin{aligned}
& \widetilde{P}(2)>0, \quad \operatorname{sign} \varepsilon \widetilde{P}(2)=\operatorname{sign} \varepsilon \quad \text { при четном } N, \\
& \widetilde{P}(2)<0, \quad \operatorname{sign} \varepsilon \widetilde{P}(2)=-\operatorname{sign} \varepsilon \quad \text { при нечетном } N \text {. }
\end{aligned}
$$

Если $\xi_{i_{0}-1}<\xi_{i_{0}}, \xi_{i_{0}}>\xi_{i_{0}+1}$ при $\varepsilon \in\left(-\varepsilon_{0}, 0\right)$ и $\xi_{i_{0}-1}>\xi_{i_{0}}, \xi_{i_{0}}<\xi_{i_{0}+1}$ при $\varepsilon \in\left(0, \varepsilon_{0}\right)$, то

$$
\begin{array}{ll}
\Phi(2, \varepsilon)>\widehat{P}(2, \widehat{\eta}) & \text { при четном } N, \\
\Phi(2, \varepsilon)<\widehat{P}(2, \widehat{\eta}) & \text { при нечетном } N .
\end{array}
$$

Полученные неравенства противоречат лемме 1. Значит, предположение (7) неверно. Следовательно, полином $\widehat{P}(\cdot, \widehat{\eta}) \in \mathfrak{P}_{N}$ и система из $N-1$ точек $\widehat{\tau}_{1}, \widehat{\tau}_{2}, \ldots, \widehat{\tau}_{N-1}$ удовлетворяют равенствам $\widehat{P}^{\prime}\left(\widehat{\tau}_{i}, \widehat{\eta}\right)=0,1 \leqslant i \leqslant N-1$. Тем самым, мы показали, что построенные по экстремальной точке $\widehat{\eta} \in \operatorname{int} \Sigma^{N-1}$ вариационной задачи 1 система точек $\widehat{\tau}=\left(\widehat{\tau}_{0}, \widehat{\tau}_{1}, \ldots, \widehat{\tau}_{N}\right)$ и полином $\widehat{P}(\cdot, \widehat{\eta}) \in \mathfrak{P}\left(\operatorname{int} \Sigma^{N-1}, \xi\right)$ удовлетворяют условиям доказываемой теоремы.

Покажем, что система точек $\widehat{\tau}=\left(\widehat{\tau}_{0}, \widehat{\tau}_{1}, \ldots, \widehat{\tau}_{N}\right) \subset \Delta$ и полином $\widehat{P}(\cdot) \in \mathfrak{P}_{N}$ условиями теоремы 1 определяются однозначно. Действительно, пусть существуют две различные системы точек $\widehat{\tau}^{1}=\left(\widehat{\tau}_{0}^{1}, \widehat{\tau}_{1}^{1}, \ldots, \widehat{\tau}_{N}^{1}\right), \widehat{\tau}^{2}=\left(\widehat{\tau}_{0}^{2}, \widehat{\tau}_{1}^{2}, \ldots, \widehat{\tau}_{N}^{2}\right)$ на отрезке $\Delta$ и два различных полинома $\widehat{P}_{1}(\cdot), \widehat{P}_{2}(\cdot) \in \mathfrak{P}_{N}$, удовлетворяющих условиям теоремы 1 . Тогда $\widehat{P}_{1}(2)=\widehat{P}_{2}(2)$, и система $\widehat{\tau}^{i}=\left(\widehat{\tau}_{0}^{i}, \widehat{\tau}_{1}^{i}, \ldots, \widehat{\tau}_{N}^{i}\right), i=1,2$, из $N+1$ точек отрезка $\Delta$ определяет точку $\widehat{\eta}^{i}=\left(\widehat{\eta}_{1}^{i}, \widehat{\eta}_{2}^{i}, \ldots, \widehat{\eta}_{N-1}^{i}\right) \in \operatorname{int} \Sigma^{N-1}$ с $N-1$ координатами $\widehat{\eta}_{j}^{i}=\widehat{\tau}_{j}^{i}-\widehat{\tau}_{j-1}^{i}$, $1 \leqslant j \leqslant N-1, i=1,2$. Будем рассматривать $\widehat{\tau}^{i}=\left(\widehat{\tau}_{0}^{i}, \widehat{\tau}_{1}^{i}, \ldots, \widehat{\tau}_{N}^{i}\right)$, как систему из 
$N+1$ точек отрезка $\Delta$, и $\widehat{P}_{i}(\cdot)$, как алгебраический полином из $\mathfrak{P}\left(\operatorname{int} \Sigma^{N-1}, \xi\right)$, построенные по точке $\widehat{\eta}^{i} \in \operatorname{int} \Sigma^{N-1}$. Тогда $\widehat{\tau}^{i}=\tau\left(\widehat{\eta}^{i}\right)=\left(\tau_{0}\left(\widehat{\eta}^{i}\right), \tau_{1}\left(\widehat{\eta}^{i}\right), \ldots, \tau_{N}\left(\widehat{\eta}^{i}\right)\right)$ и $\widehat{P}_{i}(\cdot)=\widehat{P}_{i}\left(\cdot, \widehat{\eta}^{i}\right), i=1,2$. Согласнопредположению $\widehat{\tau}^{1}$ и $\widehat{\tau}^{2}$ различные и $\widehat{P}_{1}(\cdot) \not \equiv \widehat{P}_{2}(\cdot)$, поэтому существует $i_{0}, 1 \leqslant i_{0} \leqslant N-1$, такое, что $\widehat{\tau}_{i_{0}}^{1} \neq \widehat{\tau}_{i_{0}}^{2}$. Положим

$$
\Phi(\cdot, \lambda)=\lambda \widehat{P}_{1}(\cdot)+(1-\lambda) \widehat{P}_{2}(\cdot), \quad \lambda \in \Delta .
$$

Легко показать, что при любом $\lambda \in \Delta$ полином $\Phi(\cdot, \lambda) \in \mathfrak{P}_{N}, \Phi(2, \lambda)=\widehat{P}_{i}(2), i=1,2$, и точки экстремумов $\Phi(\cdot, \lambda)$ расположены на отрезке $\Delta$. Обозначим систему этих точек через $\tau^{\lambda}=\left(\tau_{0}^{\lambda}, \tau_{1}^{\lambda}, \ldots, \tau_{N}^{\lambda}\right), 0=$ : $\tau_{0}^{\lambda}<\tau_{1}^{\lambda}<\cdots<\tau_{N}^{\lambda}:=1$. Для любого заданного числа $\varepsilon>0$ найдется число $\lambda_{0} \in(0,1)$ такое, что при всех $\lambda \in\left(0, \lambda_{0}\right)$ и $t \in \Delta$ выполнено $\left|\Phi(t, \lambda)-\widehat{P}_{1}(t)\right|<\varepsilon$. Сначала выберем положительное число $\delta$ так, чтобы $\delta$-окрестности $\left(\widehat{\tau}_{0}^{1}-\delta, \widehat{\tau}_{0}^{1}+\delta\right),\left(\widehat{\tau}_{1}^{1}-\delta, \widehat{\tau}_{1}^{1}+\delta\right), \ldots,\left(\widehat{\tau}_{N}^{1}-\delta, \widehat{\tau}_{N}^{1}+\delta\right)$ точек экстремумов $\widehat{\tau}_{0}^{1}, \widehat{\tau}_{1}^{1}, \ldots, \widehat{\tau}_{N}^{1}$ полинома $\widehat{P}_{1}(\cdot)$ попарно не пересекались. Затем подберем $\lambda_{0} \in(0,1)$ так, чтобы при любом $\lambda \in\left(0, \lambda_{0}\right)$ точка $i$-го экстремума $\tau_{i}$ полинома $\Phi(\cdot, \lambda)$ находилась в $\delta$-окрестности $\left(\widehat{\tau}_{i}^{1}-\delta, \widehat{\tau}_{i}^{1}+\delta\right)$ точки $i$-го экстремума $\widehat{\tau}_{i}^{1}$ полинома $\widehat{P}_{1}(\cdot)$. Согласно построению полиномов $\widehat{P}_{1}(\cdot), \Phi(\cdot, \lambda)$ и выбору $\lambda_{0}$ для экстремальных значений $\left\{\widehat{P}_{1}\left(\widehat{\tau}_{i}^{1}\right)\right\}_{i=0}^{N}$ и $\left\{\Phi\left(\widehat{\tau}_{i}^{1}, \lambda\right)\right\}_{i=0}^{N}$ полиномов $\widehat{P}_{1}(\cdot)$ и $\Phi(\cdot, \lambda), \lambda \in\left(0, \lambda_{0}\right)$, в точках $\left\{\widehat{\tau}_{i}^{1}\right\}_{i=0}^{N}$ экстремумов $\widehat{P}_{1}(\cdot)$ имеем

$$
\widehat{P}_{1}\left(\widehat{\tau}_{i}^{1}\right) \geqslant \Phi\left(\widehat{\tau}_{i}^{1}, \lambda\right), \quad i=0,2,4, \ldots, \quad \widehat{P}_{1}\left(\widehat{\tau}_{i}^{1}\right) \leqslant \Phi\left(\widehat{\tau}_{i}^{1}, \lambda\right), \quad i=1,3,5, \ldots,
$$

если $\widehat{P}_{1}\left(\widehat{\tau}_{0}^{1}\right)>\widehat{P}_{1}\left(\widehat{\tau}_{1}^{1}\right)$, или

$$
\widehat{P}_{1}\left(\widehat{\tau}_{i}^{1}\right) \leqslant \Phi\left(\widehat{\tau}_{i}^{1}, \lambda\right), \quad i=0,2,4, \ldots, \quad \widehat{P}_{1}\left(\widehat{\tau}_{i}^{1}\right) \geqslant \Phi\left(\widehat{\tau}_{i}^{1}, \lambda\right), \quad i=1,3,5, \ldots,
$$

если $\widehat{P}_{1}\left(\widehat{\tau}_{0}^{1}\right)<\widehat{P}_{1}\left(\widehat{\tau}_{1}^{1}\right)$. Поэтому разность $\widehat{P}_{1}(t)-\Phi(t, \lambda), \lambda \in\left(0, \lambda_{0}\right)$, имеет не менее $N+2$ нулей: по одному нулю в каждой из $N+1$ попарно не пересекающихся $\delta$-окрестностей $\left\{\left(\widehat{\tau}_{i}^{1}-\delta, \widehat{\tau}_{i}^{1}+\delta\right)\right\}_{i=0}^{N}$ точек $\left\{\widehat{\tau}_{i}^{1}\right\}_{i=0}^{N}$ и один нуль в точке $t=2$. Получили противоречие с тем, что $\widehat{P}_{1}(t)-\Phi(t, \lambda) \not \equiv 0, \lambda \in\left(0, \lambda_{0}\right)$, есть алгебраический полином степени $N$. Значит, наше предположение о существовании двух различных систем $\left(\widehat{\tau}_{0}^{1}, \widehat{\tau}_{1}^{1}, \ldots, \widehat{\tau}_{N}^{1}\right)$, $\left(\widehat{\tau}_{0}^{2}, \widehat{\tau}_{1}^{2}, \ldots, \widehat{\tau}_{N}^{2}\right)$ и полиномов $\widehat{P}_{1}(\cdot), \widehat{P}_{2}(\cdot)$, удовлетворяющих условиям теоремы 1 , неверно. Поэтому $\widehat{\tau}_{i}^{1}=\widehat{\tau}_{i}^{2}, 0 \leqslant i \leqslant N$. Следовательно, $\widehat{\eta}_{i}^{1}=\widehat{\eta}_{i}^{2}, 0 \leqslant i \leqslant N-1$, и $\widehat{P}_{1}(\cdot)=\widehat{P}_{2}(\cdot)$. Таким образом, мы показали, что существуют единственный алгебраический полином $\widehat{P}(\cdot) \in \mathfrak{P}_{N}$ и единственная система из $N+1$ точек $\left(\widehat{\tau}_{0}, \widehat{\tau}_{1}, \ldots, \widehat{\tau}_{N}\right)$ на отрезке $\Delta$, удовлетворяющие условиям доказьваемой теоремы. Теорема 1 доказана.

СлЕДСтВИЕ 1. Задача 1 имеет единственное решение $\widehat{\eta} \in \operatorname{int} \Sigma^{N-1}$.

СледСТВИЕ 2. В классе алгебраических полиномов $\mathfrak{P}\left(\operatorname{int} \Sigma^{N-1}, \xi\right)$ әкстремальное значение $\lambda_{N}(\xi)$ задачи 1 реализуется на единственном полиноме $\widehat{P}(\cdot) \in$ $\mathfrak{P}\left(\right.$ int $\left.\Sigma^{N-1}, \xi\right)$, удовлетворяющем условиям теоремы 1.

ДоКАЗАТЕЛЬСТВо ТЕОРЕМЫ 2. Построенные по решению $\widehat{\eta}^{\prime}=\left(\widehat{\eta}_{1}, \widehat{\eta}_{2}, \ldots, \widehat{\eta}_{N-1}\right)$ задачи 2 система из $N+1$ точек $\widehat{\tau}^{\prime}=\left(\tau_{0}\left(\widehat{\eta}^{\prime}\right), \tau_{1}\left(\widehat{\eta}^{\prime}\right), \ldots, \tau_{N}\left(\widehat{\eta}^{\prime}\right)\right)=:\left(\widehat{\tau}_{0}^{\prime}, \widehat{\tau}_{1}^{\prime}, \ldots, \widehat{\tau}_{N}^{\prime}\right)$ и сплайн $x\left(\cdot, \hat{\eta}^{\prime}\right) \in S_{m}\left(\Delta, R_{n}\right)$ удовлетворяют условиям теоремы 2 . Действительно, в силу построения $\widehat{\tau}^{\prime}$ и $x\left(\cdot, \widehat{\eta}^{\prime}\right)$ имеем

$$
\begin{gathered}
0=: \widehat{\tau}_{0}^{\prime}<\widehat{\tau}_{1}^{\prime}<\cdots<\widehat{\tau}_{N-1}^{\prime}<\widehat{\tau}_{N}^{\prime}:=1, \\
x\left(\widehat{\tau}_{i}^{\prime}, \widehat{\eta}^{\prime}\right)=\xi_{i}, \quad 0 \leqslant i \leqslant N,
\end{gathered}
$$


так как $\widehat{\eta}^{\prime} \in \operatorname{int} \Sigma_{R_{n}}^{N-1}$ и $x\left(\cdot, \widehat{\eta}^{\prime}\right) \in S\left(\operatorname{int} \Sigma_{R_{n}}^{N-1}, \xi\right)$.

Допустим, что в точке $\widehat{\tau}_{j 0}^{\prime}, 1<j_{0}<N$, нарушено хотя бы одно из равенств (2), т.е.

$$
x^{\prime}\left(\widehat{\tau}_{j_{0}}^{\prime}, \widehat{\eta}^{\prime}\right) \neq 0
$$

Сплайн $s_{j_{0}}(t) \in S_{m}\left(\Delta, R_{n}\right)$, полученный из сплайна $(6)$ при $i=j_{0}$, имеет максимальное число $N$ нулей в точках $\widehat{\tau}_{0}^{\prime}, \widehat{\tau}_{1}^{\prime}, \ldots, \widehat{\tau}_{j_{0}-1}^{\prime}, \widehat{\tau}_{j_{0}+1}^{\prime}, \ldots, \widehat{\tau}_{N}^{\prime}$ отрезка $\Delta$, поэтому его значение $s_{j_{0}}(2)$ в точке $t=2$ отлично от 0 , т.е. $s_{j_{0}}(2) \neq 0$. Тогда согласно теореме о неявной функции [22, с. 166] существуют $\varepsilon_{0}>0$ и отображение $T(\varepsilon) \in C^{1}\left[-\varepsilon_{0}, \varepsilon_{0}\right]$ такие, что $T(0)=\widehat{\tau}_{j_{0}}^{\prime}$ и $x\left(T(\varepsilon), \widehat{\eta}^{\prime}\right)+\varepsilon s_{j_{0}}(T(\varepsilon))=\xi_{j_{0}}$ при любом $\varepsilon \in\left(-\varepsilon_{0}, \varepsilon_{0}\right)$. Отсюда следует, что при любом $\varepsilon \in\left(-\varepsilon_{0}, \varepsilon_{0}\right)$ сплайн $\Psi(t, \varepsilon):=x\left(t, \widehat{\eta}^{\prime}\right)+\varepsilon s_{j_{0}}(t) \in S\left(\operatorname{int} \Sigma_{R_{n}}^{N-1}, \xi\right)$. Исследуем значение $\Psi(t, \varepsilon)$ в точке $t=2$. Заметим, что

$$
\begin{aligned}
& s_{j_{0}}(2)>0, \quad \operatorname{sign} \varepsilon s_{j_{0}}(2)=\operatorname{sign} \varepsilon \quad \text { при четном } N, \\
& s_{j_{0}}(2)<0, \quad \operatorname{sign} \varepsilon s_{j_{0}}(2)=-\operatorname{sign} \varepsilon \quad \text { при нечетном } N \text {. }
\end{aligned}
$$

Если $\xi_{i_{0}-1}<\xi_{i_{0}}, \xi_{i_{0}}>\xi_{i_{0}+1}$ при $\varepsilon \in\left(0, \varepsilon_{0}\right)$, то

$$
\begin{array}{ll}
\Psi(2, \varepsilon)>x\left(2, \widehat{\eta}^{\prime}\right) & \text { при четном } N, \\
\Psi(2, \varepsilon)<x\left(2, \widehat{\eta}^{\prime}\right) & \text { при нечетном } N .
\end{array}
$$

Полученные неравенства противоречат теореме 2. Значит, предположение (9) неверно. Поэтому сплайн $x\left(\cdot, \widehat{\eta}^{\prime}\right) \in S_{m}\left(\Delta, R_{n}\right)$ и система из $N-1$ точек $\left(\widehat{\tau}_{1}^{\prime}, \widehat{\tau}_{2}^{\prime}, \ldots, \widehat{\tau}_{N-1}^{\prime}\right)$ удовлетворяют равенствам $x^{\prime}\left(\widehat{\tau}_{i}^{\prime}, \widehat{\eta}^{\prime}\right)=0,1 \leqslant i \leqslant N-1$. Таким образом, мы показали, что построенные по экстремальной точке $\widehat{\eta}^{\prime} \in$ int $\Sigma_{R_{n}}^{N-1}$ вариационной задачи 2 система из $N+1$ точек $\widehat{\tau}^{\prime}=\left(\widehat{\tau}_{0}^{\prime}, \widehat{\tau}_{1}^{\prime}, \ldots, \widehat{\tau}_{N}^{\prime}\right)$ и сплайн $x\left(\cdot, \widehat{\eta}^{\prime}\right) \in S\left(\operatorname{int} \Sigma_{R_{n}}^{N-1}, \xi\right)$ удовлетворяют условиям теоремы 2 .

Единственность системы точек $\widehat{\tau}^{\prime}=\left(\widehat{\tau}_{0}^{\prime}, \widehat{\tau}_{1}^{\prime}, \ldots, \widehat{\tau}_{N}^{\prime}\right) \subset \Delta$ и сплайна $\widehat{x}(\cdot) \in S_{m}\left(\Delta, R_{n}\right)$, удовлетворяющих условиям теоремы 2 , докажем от противного. Допустим, что существуют две различные системы точек $\widehat{\tau}^{\prime 1}=\left(\widehat{\tau}_{0}^{\prime 1}, \widehat{\tau}_{1}^{\prime 1}, \ldots, \widehat{\tau}_{N}^{\prime 1}\right), \widehat{\tau}^{\prime 2}=\left(\widehat{\tau}_{0}^{\prime 2}, \widehat{\tau}_{1}^{\prime 2}, \ldots, \widehat{\tau}_{N}^{\prime 2}\right)$ на отрезке $\Delta$ и два различных сплайна $\widehat{x}_{1}(\cdot), \widehat{x}_{2}(\cdot) \in S_{m}\left(\Delta, R_{n}\right)$, для которых выполнены условия теоремы 2 . Рассмотрим сплайн $\Phi^{\prime}(\cdot, \lambda):=\lambda \widehat{x}_{1}(\cdot)+(1-\lambda) \widehat{x}_{2}(\cdot) \in S_{m}\left(\Delta, R_{n}\right)$ при любом $\lambda \in \Delta$. Повторяя дословно рассуждения, проведенные для полинома (8) при доказательстве теоремы 1 , покажем, что $\widehat{\tau}_{i}^{\prime 1}=\widehat{\tau}_{i}^{\prime 2}, 0 \leqslant i \leqslant N$, и $\widehat{x}_{1}(\cdot)=\widehat{x}_{2}(\cdot)$. Тем самым, теорема 2 доказана.

СлЕдСтВиЕ 3. Задача 2 имеет единственное решение $\widehat{\eta}^{\prime} \in \operatorname{int} \Sigma_{R_{n}}^{N-1}$.

СлЕдСТВИЕ 4. Среди сплайнов из $S\left(\operatorname{int} \Sigma_{R_{n}}^{N-1}, \xi\right)$ әкстремальное значение $\lambda_{N}^{\prime}(\xi)$ задачи 2 реализуется на единственном сплайне $\widehat{x}(\cdot) \in S\left(\operatorname{int} \Sigma_{R_{n}}^{N-1}, \xi\right)$, удовлетворяющем условиям теоремы 2.

ЗАмечаниЕ. Разные обобщения теоремы 1 , полученные в работах [11]-[14], [20], [22]-[24] различньми методами, доказьваются подобно теоремам 1 и 2.

Автор выражает искреннюю благодарность научному руководителю В. М. Тихомирову за постановку задачи, постоянное внимание и помощь, а также рецензенту за замечания, которые способствовали улучшению настоящей статьи. 


\section{СПИСОК ЦИТИРОВАННОЙ ЛИТЕРАТУРЫ}

[1] Иоффе А. Д., Тихомиров В. М. Теория экстремальных задач. М.: Наука, 1974.

[2] Алексеев В. М., Галеев Э. М., Тихомиров В. М. Сборник задач по оптимизации. М.: Наука, 1984.

[3] Тихомиров В. М. Некоторые вопросы теории приближений. М.: Изд-во МГУ, 1976.

[4] Davis Ch. Problem 4714 // Amer. Math. Monthly. 1956. V. 63. № 10. P. 729.

[5] Davis Ch. Extrema of a polynomial // Amer. Math. Monthly. 1957. V. 64. №9. P. 679-680.

[6] Kempner A. J. On the shape of polynomial curves // Tôhoku Math. J. 1933. V. 37. P. 347-362.

[7] Kempner A. J. On the shape of polynomial curves. II // Tôhoku Math. J. 1936. V. 42. P. $318-330$.

[8] Mycielski J., Paszkowski S. A generalization of Chebyshev polynomials // Bull. Acad. Polon. Sci. Ser. Math. Astron. Phys. 1960. V. 8. P. 433-438.

[9] Paszkowski S. The Theory of Uniform Approximation. I: Non-Asymptotic Theoretical Problems. Rosprawy Mathematyczne. V. 26. Warszawa, 1962.

[10] Kammerer W. J. Polynomial approximations to finitely oscillating functions // Math. Comp. 1961. V. 15. P. 115-119.

[11] Виденский В. С. Об одном классе интерполяционных многочленов с незакрепленными узлами // Докл. АН СССР. 1965. Т. 162. № 2. С. 251-254.

[12] Виденский В.С. Существование и единственность решения одной интерполяционой задачи // Сб. научн. тр. Ленинградского механического института. Т. 50. Ленинград: ЛМИ, 1965. C. $29-41$.

[13] Виденский В. С. О построении многочлена по данной последовательности его экстремумов // Сб. статей "Современные проблемы теории аналитических функций". Труды международной конференции по теории аналитических функций (Ереван, 1965). М.: Наука, 1966. С. 62-64.

[14] Виденский В. С. Теорема существования многочлена с данной последовательностью экстремумов // Докл. АН СССР. 1966. Т. 171. № 1. С. 17-20.

[15] Kudela J. Construction des certains polynômes extrémaux // Zeszyty Nauk. Uniw. Jagielloń. Prace Mat. 1963. V. 77. № 9. P. 31-36.

[16] Коробкова М. Б. Теорема существования сплайн-полинома с данной последовательностью экстремумов // Матем. заметки. 1972. Т. 11. № 3. С. 251-258.

[17] Johnson R.S. On monosplines of least deviation // Trans. Amer. Math. Soc. 1960. V. 96. P. 458-477.

[18] Завьялов Ю. С., Квасов Б. И., Мирошниченко В. Л. Метод сплайн-функций. М.: Наука, 1980.

[19] Schoenberg I. J., Whitney A. On Polya frequency functions. III. The positivity of translation determinants with an application to the interpolation problem by spline curves // Trans. Amer. Math. Soc. 1953. V. 74. P. 246-259.

[20] Дэйвис Ч. Свойства отображений некоторых систем Чебышева // Докл. АН СССР. 1967. Т. 175 . № 2. C. $280-283$.

[21] Алексеев В. М., Тихомиров В. М., Фомин С. В. Оптимальное управление. М.: Наука, 1979.

[22] Fitzgerald C. H., Schumaker L. L. A differential equation approach to interpolation at extremal points // J. Anal. Math. 1969. V. 22. P. 117-134.

[23] Виденский В. С. Обобщение некоторых теорем о системах Чебышева на интерполяционные классы // Сб. трудов ЛГПИ им. А. И. Герцена. Л., 1972. С. 45-67.

[24] Bojanov B.D. A generalization of Chebyshev polynomials // J. Approx. Theory. 1979. V. 26. P. 293-300. 